\title{
The Solar Neutrino Problem Has Not Been Solved
}

\section{ABSTRACT}

\author{
Jamal S. Shrair \\ Helical Universe \\ www.helical-universe.info \\ helical.cosmos@gmail.com
}

A closer look at the data collected from different detectors, reveal that the so-called solar neutrino problem is far from being solved. And contrary to the assessment of the Nobel Committee, the experimental results from the Sudbury Neutrino Observatory cannot be regarded as a confirmation of the Standard Solar Model. In fact, the obsoleteness of the current model has been recently exposed by the crisis of solar abundance. Furthermore, using images obtained by the Solar Dynamics Observatory, researchers found the convective motions (the plasma motions within the Sun's interior) to be nearly 100 times smaller than current theoretical expectations. This is a clear verdict on the notion of thermonuclear reactions that are supposed to be the processes that generate solar neutrinos.

\section{KEYWORDS}

Solar neutrino problem, neutrino oscillations, neutrino detectors, solar physics, standard solar model, astrophysics,

\section{ACADEMIC DISCIPLINE AND SUB-DISCIPLINES}

Solar physics and Astrophysics

\section{SUBJECT CLASSIFICATION}

Fundamental Solar Physics and Astrophysics

\section{TYPE (METHOD/APPROACH)}

Quantitative and Qualitative

\section{INTRODUCTION}

Based on the hypothesis of the standard solar model, the number of neutrinos produced by the Sun per second is approximately $1.8 \times 10^{38}$, which means 400 trillion neutrinos pass through our bodies every second. The model, also assumes that the absolute majority of neutrinos is, generated from the P-P reaction, and $99.99 \%$ of solar neutrinos have energy which is far too low for detection. Solar neutrinos with higher energy are considered to be rare, making up merely $0.01 \%$ of solar neutrinos. These high energy neutrinos come from a side reaction involving $3 \mathrm{He}$ and $4 \mathrm{He}$ particles that form a beryllium nucleus (7Be) which then captures a proton to form a boron nucleus (8B). This nucleus then breaks up into Beryllium $(8 \mathrm{Be})$ plus a positron and neutrino. Furthermore, just two of these reactions are supposed to be produced out of 10,000 completions of the P-P reaction. In order to detect these high energy neutrinos, it was first proposed by Raymond Davis Jr that a vessel, about the size of a large swimming pool (400 cubic meters) filled with a chlorine-based cleaning fluid (Perchloroethylene) was placed underground in a gold mine in South Dakota, USA. Raymond Davis Jr used a chlorine solution as a target for inverse $\beta$-interactions, $\mathbf{v}_{\mathbf{e}}+{ }^{37} \mathbf{C l} \rightarrow{ }^{37} \mathbf{A r}+\mathbf{e}^{-}$. The radioactive ${ }^{37} \mathbf{A r}$ could be extracted from the target and the decay could be detected with proportional counters. It is believed only a small number of the Chlorine-37 $(37 \mathrm{Cl})$ will react with the neutrinos to form Argon-37 and an electron, which then reverts to Chlorine-37 and neutrino. (37Ar $+\mathrm{e}^{-} \rightarrow \mathbf{3 7 C l}+\mathrm{v}$ e) The $37 \mathrm{Ar}$ atoms are purged with helium gas and the decay is counted. The Homestake Solar Neutrino Detector was only sensitive to neutrinos with energy higher than $\sim \mathbf{0 . 8} \mathbf{M e V}$. The first results of Ray's experiment were announced in 1968 (1). The detector should have measured $8 \times 10^{36}$ interactions per second per atom or $\mathbf{8 S N U}$, with an error rate of $33 \%$. 


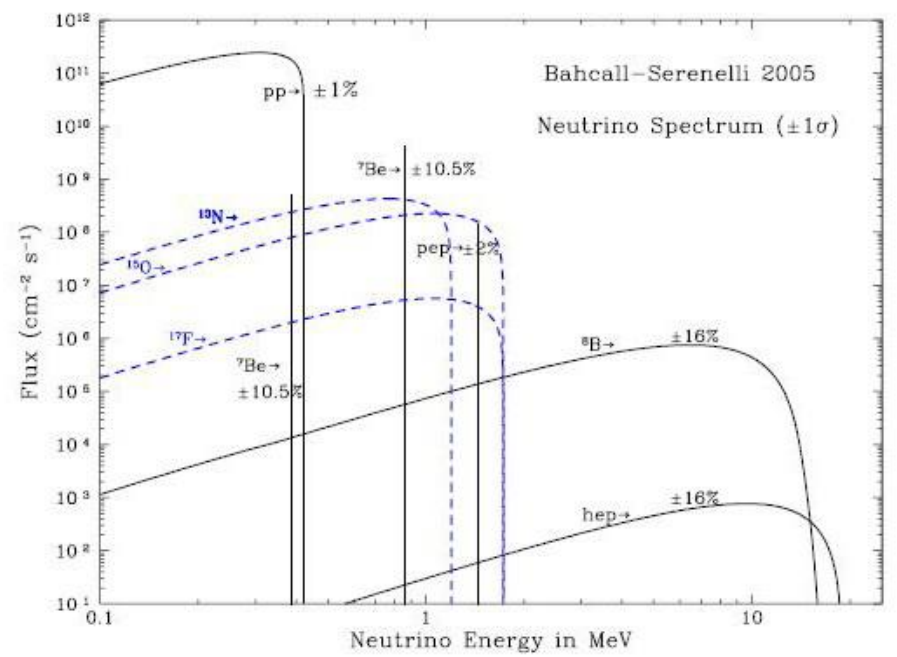

Figure 1.0 Calculated energy spectrum of the solar neutrinos (with 1 standard deviation uncertainty)
according to the Standard Solar Model [1]

However, the neutrino detector had averaged just 2.0 SNU with a deviation of 0.3 SNU. This represented about one third as many radioactive Argon atoms as were predicted. The discrepancy is well outside both the uncertainty of the calculations and the experimental deviations. This discrepancy soon became known as "The solar neutrino problem" or "The mystery of the missing neutrinos". In the beginning two different explanations were given to solve this problem, but, no question was raised about the validity of thermonuclear powered Sun. The first explanation, suggested that the theoretical calculations might be wrong. The second explanation suggested that the set up of the experiment could be wrong. For the following two decades, the sensitivity of the experiment was increased and the theoretical calculations were refined and checked many times. In spite of that the discrepancy between the theory and experimental results persisted.

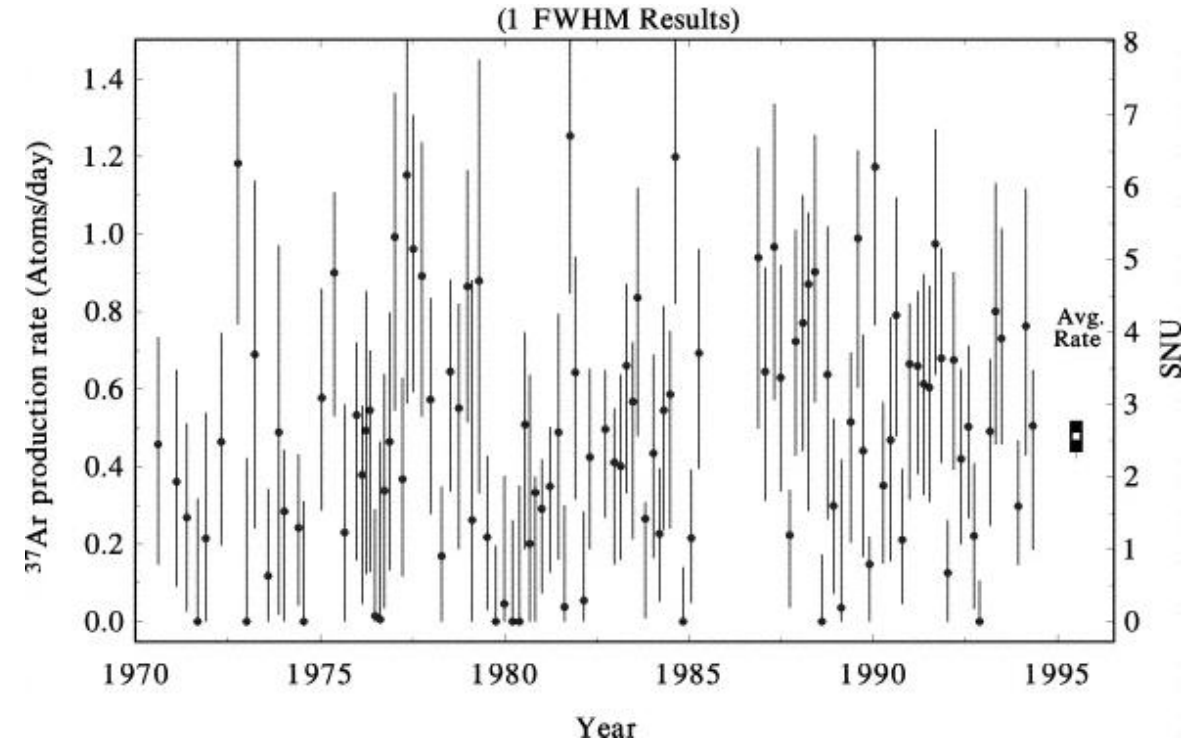

Figure 2.0 the measured flux of solar neutrinos by Homestake Solar Neutrino Detector (1970-1995)

Twenty-one years after the results of Davis's experiment were announced, a new experimental group (JapaneseAmerican group) called Kamiokande led by two Japanese, Masatoshi Koshiba and Yoji Totsuka constructed a large pure water detector, in order to measure the rate at which electrons in the water scattered the higher energy neutrinos that are supposed to be emitted from the Sun, and considered to be rare in the solar energy production cycle. The results of Kamiokande also showed the neutrino events observed were far less than those predicted by the theoretical model. The discrepancy between the theory and experiment remains, although in the case of Kamiokande the discrepancy was slightly less than in the chlorine detector of Ray Davis. In the 1990s, three other new and massive detectors were built which also confirmed the results of the above-mentioned detectors. Two of those detectors that contained gallium were constructed in Russia and Italy. SAGE detector was led by Russian Vladimir Gavrin and GALLEX detector was led by the German Till Kirsten (4). The results of those two detectors also showed that lower energy neutrinos were missing. In addition to, SAGE and GALLEX, a much larger version of the Japanese water detector, called Super-Kamiokande directed by Totsuka and Suzuki, carried out measurements of the higher energy neutrinos and confirmed the deficit of higher energy neutrinos that were found previously by Kamiokande and Ray Davis's detector (5). 

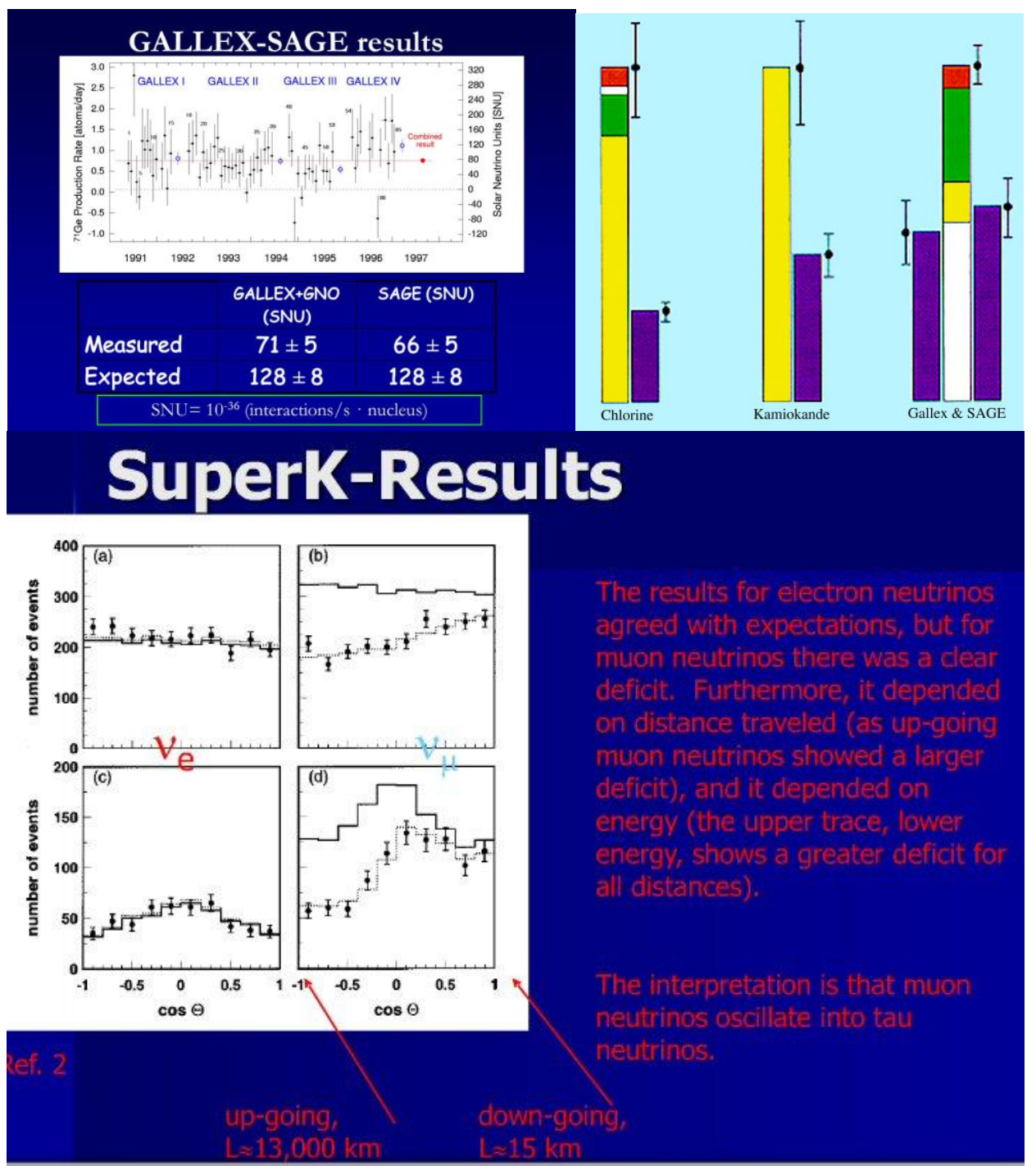

Figure 3.0 on the upper right. SOLAR NEUTRINO FLUXES observed (purple) by the four first-generation detectors show large deficits when compared with standard solar-model predictions. The different predicted total fluxes, normalized here to unity, comprise various individual solar processes, indicated by colors: white for pep fusion (with and without electron assistance); green for electron capture by 7Be; yellow for 8B decay; and red for the stellar carbon-nitrogen-oxygen cycle, which is calculated to play only a small role in the Sun neutrinos so energetic that Kamiokande can see them. The monoenergetic line fluxes are shown in cm-2.s-1. The secondgeneration water-Cerenkov experiments hope to have neutrino-energy detection thresholds of about 5 MeV. (Credit: John N. Bahcall, Frank Calaprice,Arthur B. McDonald and Yoji Totsuka, Physics Today, Volume 49, No. 7, July 1996, pp. 30-36)

\section{DISCUSSION \& ANALYSIS}

It is abundantly clear from the results of the above mentioned detectors that the expected number of both high and low energy neutrinos were absent. The huge discrepancy between the expectations of the model and the experimental results remained in spite of decades of trying with different detectors. In other words, all detectors showed that the number of neutrinos is far too few to account for the Sun's presumed thermonuclear energy output. Theoreticians had to find a solution to save their model, because they cannot contemplate trashing the theory that resides at the heart of astrophysics and cosmology. Therefore, the problem had to be with the neutrinos themselves. As expected theoreticians created a hypothetical problem for solar neutrinos, but this time the Standard Model of Particle physics has to be scarified, yet, the model is supposed to be verified by so many experiments that took enormous amounts of time and money $(2,3)$.

However, theoretical physicists came up with the idea that solar neutrinos have a multiple personality disorder just like so many politicians. The notion assumes that neutrinos are created as electron neutrinos in the Sun, but during their journey to Earth they change their type. And of course this theoretical notion has to be backed up or supported by some types of experimental evidence. On June 18, 2001 a collaboration of Canadian, American, and British scientists directed by the Canadian scientist Arthur B. McDonald announced that the solar neutrino mystery had been solved. They used a detector that contains 1000 tons of heavy water $\left(\mathrm{D}_{2} \mathrm{O}\right)$. The detector is called Solar Neutrino Observatory (SNO) located in a nickel mine in Sudbury, Ontario, Canada. The researchers stated that they studied the same higher-energy neutrinos that had been investigated previously in Japan with the Kamiokande and Super-Kamiokande detectors. The first measurement was 
performed by using the heavy-water detector in a "mode" which is sensitive only to electron neutrinos. They obtained around one-third as many neutrinos as the standard solar model predicted. The researchers combined their results with the previous results of Kamiokande and Super-Kamiokande detectors and announced that the solar neutrino problem had now been solved.
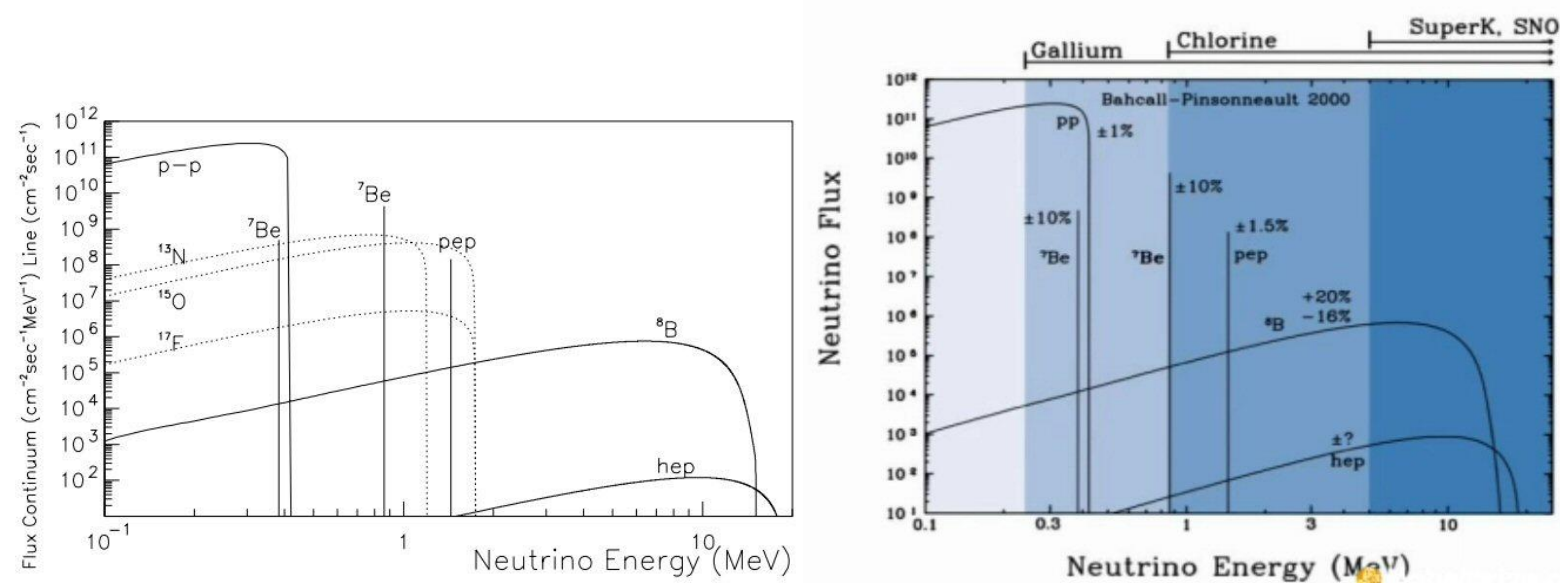

Figure 4.0 Solar neutrino fluxes --- pp chain (solid lines) (dotted lines are CNO neutrinos, which is less than $2 \%$ of total). right -- superimposes the neutrino detection threshold of the major neutrino detectors (source left) http://www.slac.stanford.edu/econf/C990809/docs/suzuki.pdf) (source right) -- screen capture from 2002 Davis Nobel lecture)

Based on their assessment the combined measurements of the SNO and the two Japanese detectors agreed with the prediction of the standard solar model. In their first measurement which is supposed to be sensitive only to electron neutrinos, the SNO researchers said, they observed approximately one-third as many electron neutrinos as the model predicted. They concluded that since the Super-Kamiokande detector, which is sensitive to electron neutrinos as well as to the other types, observed about half as many events as were expected, thus the smoking gun has been found.

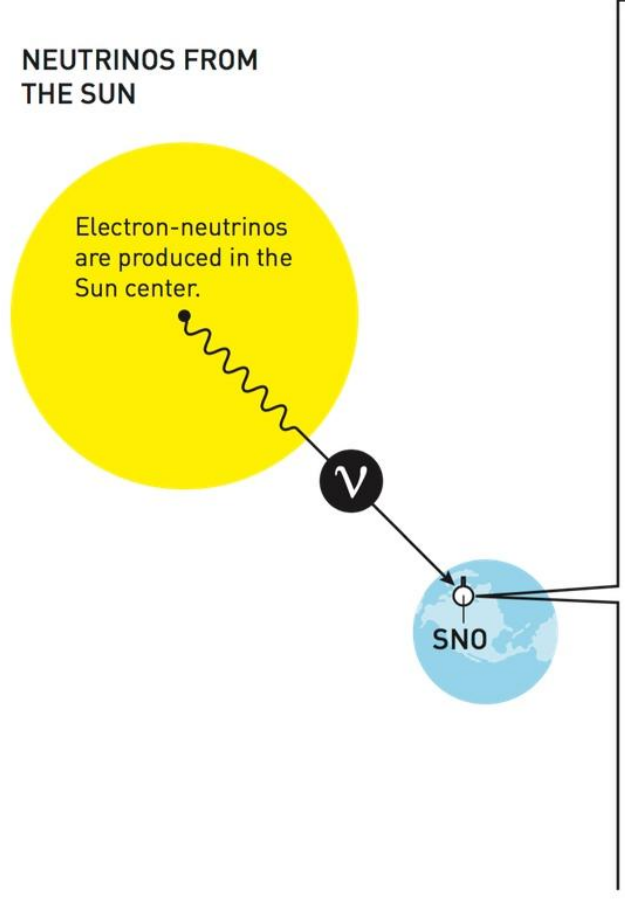

SUDBURY NEUTRINO OBSERVATORY (SNO)

ONTARIO, CANADA

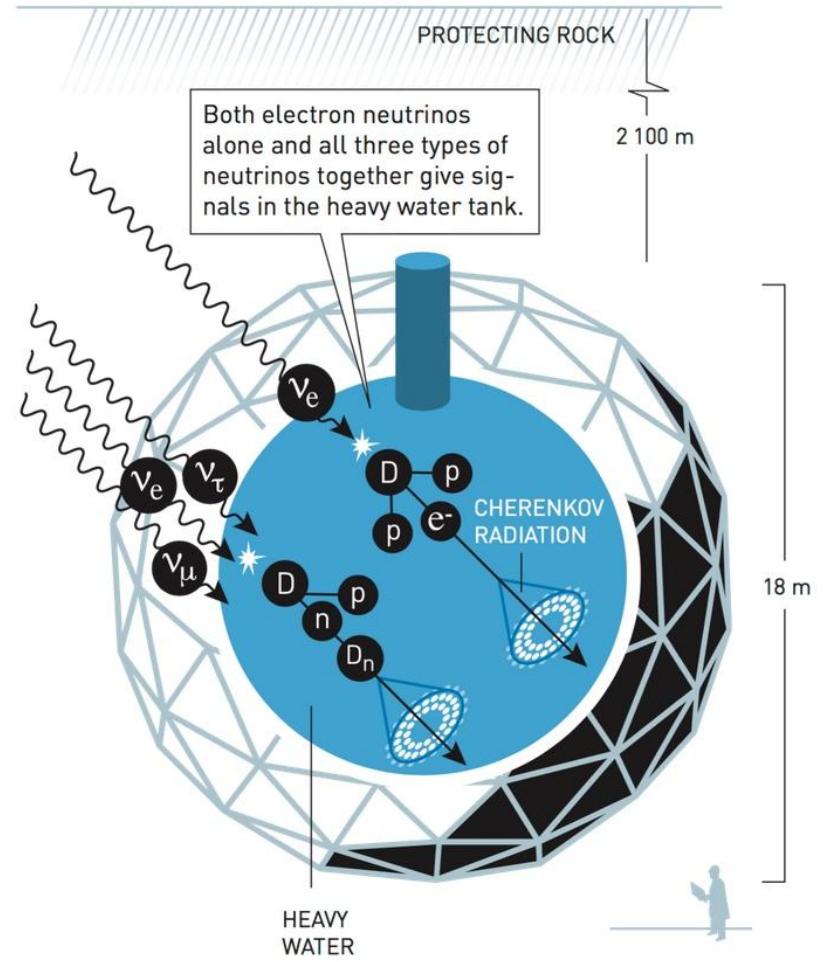

Figure 5.0 Credit: Johan Jarnestad/the Royal Swedish Academy of Sciences. 


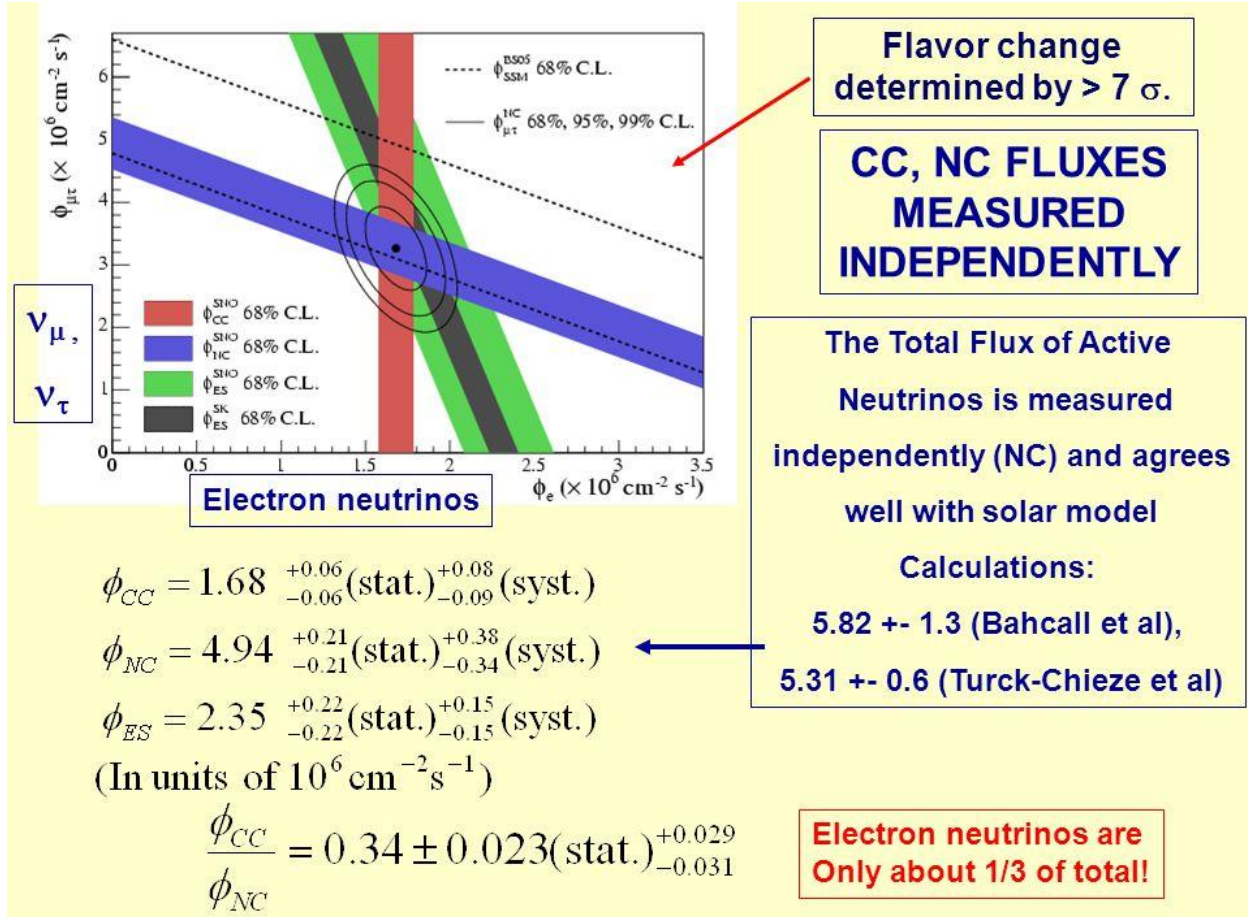

Figure 6.0 Credit: Arthur B. McDonald, Queen's University, Kingston, Ontario, Canada

In the above figure obtained from the research team of SNO, the x-axis representing electron-neutrino flux, while the $y$ axis represents the flux of the other types. Yet, it is not possible to distinguish muon neutrinos from tau neutrinos. The red band shows the result of the charged-current analysis, which is sensitive only to electron-neutrinos. The blue band is the neutral-current analysis, which is supposed to be sensitive to the three types. The green band is elastic electron-neutrino scattering, which also has some sensitivity to tau and muon neutrinos. The brown band represents the result from Super$\mathrm{K}$. The band between the dotted lines is the total neutrino flux as expected from the standard solar model. However, Bahcall and his colleagues announced that particle physics must be wrong concerning neutrinos. They claimed if particle physics was correct then the fraction measured by SNO and the fraction measured by Super-K should be the same, and all neutrinos should be electron neutrinos. Therefore, since the fractions were different, that means electron neutrinos that are produced in "the core of the Sun" change into muon and tau neutrinos by the time they reach the Earth. In other words, the missing neutrinos were actually present, but they were more difficult to be detected since they became muon and tau neutrinos.

Total Rates: Standard Model vs. Experiment Bahcall-Pinsonneault 2000

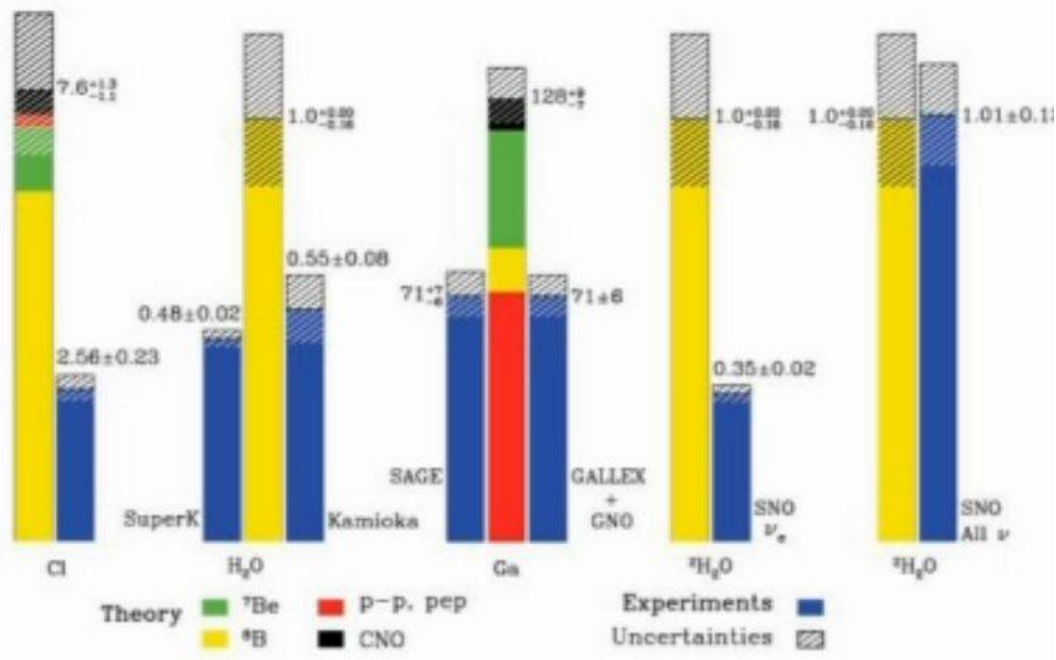

Figure 7.0 Measured (blue) vs calculated (yel) neutrino rates for all major neutrino detectors up to year 2000. Only Sudbury (SNO) in its deuterium fission mode (right) is "supposed" to show the measured rate matching the calculated rate. (screen capture from Davis Homestake 2002 Nobel lecture) 
A report in Physics Today, March 2003, stated: "After 36 years of solar neutrino experiments, the inescapable conclusion is that a large fraction of the electron neutrinos produced by nuclear processes in the Sun's core are metamorphosing into other neutrino varieties somewhere en route to the detectors on Earth." The best comment on this report came from Wal Thornhill, a dissident scientist. He said, "The report came to the conclusion that neutrinos were not undergoing any significant change of flavor in the vacuum of space between the Sun and Earth. Instead they were performing "an irreversible flavor change that takes place in high-density regions of the Sun." So not only does the Sun need a hypothetical hot, high-density core to have any hope of generating thermonuclear energy, it now needs a hypothetical "critical-electron-density region" as well, to fudge the neutrino results." On October 11, 2002, a few days after Ray Davis and Masatoshi Koshiba were awarded the Nobel Prize, Chen-Ning Yang, who received the 1957 Nobel Prize in Physics, said that "I did not believe in neutrino oscillations even after Davis' painstaking work and Bahcall's careful analysis. The oscillations were, I believed, uncalled for." Sidney Drell, an American theoretical physicist and arms control expert, who is also professor emeritus at the Stanford Linear Accelerator Center (SLAC), wrote a letter to John. N. Bahcall who worked on the solar neutrino problem for years and supported neutrino oscillations. "The success of the Standard Model of Particle Physics (SMPP) was too dear to give up. The SMPP is a beautiful theory that has been tested and found to make correct predictions for thousands of laboratory experiments. The standard solar model involves complicated physics in unfamiliar conditions and had not previously been tested to high precision. Moreover, the predictions of the standard solar model depend sensitively on details of the model, such as the central temperature. No wonder it took scientists a long time to blame the SMPP rather than the standard solar model ".

However, according to, Pontecorvo and Gribov-who were the first to come up with the idea of neutrino oscillation-lower energy solar neutrinos switch from electron neutrino to other type as they travel in the vacuum from the Sun to the Earth. Meanwhile, Stas Mikheyev, Alexei Smirnov, and Lincoln Wolfenstein proposed that the interactions of high energy neutrinos with the electrons in the Sun exacerbate their personality disorder (6). "The interactions of high energy neutrinos with the electrons in the Sun exacerbate their personality disorder, $i$.e. the presence of matter would cause the neutrinos to oscillate more vigorously between different types". On the other hand, the standard model of particle physics, asserts that there are three different types of neutrinos, one for each charged lepton, and three antineutrinos. All the three neutrinos have zero mass and they are left-handed, while the antineutrinos are right-handed. Moreover, the model assumes that these particles travel with a velocity close to that of light and they pass through matter undisturbed (or rather interact very weakly with matter). So, why then does the neutrino change its characteristic completely by converting to different types when it propagates from the core of the Sun to the surface of the Earth? This is a serious contradiction.

More important, in experimental science, one has to carry out measurements not making a hypothesis. There can be no confirmation of neutrino oscillation between the Sun and the Earth without simultaneous neutrino measurements being made near the Sun or at least somewhere in the middle between the two celestial bodies. In fact, even if one uses the arguments of those theoreticians who came up with the idea of neutrino oscillation, the solar neutrino problem is far from being solved. First of all, the mass of any particular neutrino is not specified, but only the sum of the masses of the three neutrinos is assumed to be less than 1.5 electron Volts. Neutrino oscillation requires that the three neutrinos have finite and different mass, which is still unverified. Secondly, the claim of neutrino oscillation is not backed up with any evidence showing that neutrinos oscillate routinely into any special alternate form. Neutrino oscillation is only based on theoretical hypothesis, by assuming upper limits on the oscillation rate, the so-called mixing angles. Beside that those theoreticians cannot decide whether neutrinos are Majorana particles (fermions) or Dirac particles, and why only neutrinos oscillate among leptons? On the top of all that, even if one accepts for the sake of argument that neutrinos oscillate and change flavors this is still cannot be a confirmation of the standard model. The water detectors are sensitive only to higher energy neutrinos (with energy $>5 \mathrm{eV}$ ). These neutrinos are supposed to make only $0.01 \%$ of the neutrinos emitted by the Sun. That means $99.99 \%$ of the neutrinos generated by the Sun-according to the SSM- are not detected yet. So how can one make a conclusion from these results, not to mention proclaiming that these results, agree with the theoretical predictions of the model. Precise measurements of the rate of low-energy solar neutrinos remain to be the critical test of the standard solar model and the theory of stellar evolution in general.

Nevertheless, from the analysis of data, collected by different detectors for more than three decades, it is obvious that just like in the case of quantum tunneling that supposed to remove the hurdle facing thermonuclear reactions, neutrino oscillations which is also a notion based on quantum mechanics got shoved down our throats by the help of quasiexperiment in order to be a solution to one of the most obvious defects of the theory which is a major theoretical paradigm in astrophysics. It is noteworthy, however, that the rate of neutrino production is directly related to the notion of Quantum Tunneling (QT). That is because the number of charged particles (P-P) that must collide to generate the reaction is small, compared to the total number of protons. In other words, only a tiny fraction of the nuclear collisions in the sun can overcome the potential barrier and cause the reactions. This fraction is so sensitive to temperature. Just $1 \%$ error in the temperature corresponds to about 30\% error in the predicted number of neutrinos, and $3 \%$ error in the temperature results in an error of factor of two. Recent observations using sensitive imaging devices, on board advanced space telescope, Solar Dynamic Observatory (SDO) have shown that the motions of the plasma within the interior of the Sun to be around two orders of magnitude slower than what theoretical models predict. This recent observational data is clear verdict on the real temperature range within the core of the sun and that means the hypothetical (QT) cannot possibly take place under such a condition. 


\section{CONCLUSION AND FUTURE WORK}

Our star is misunderstood, and the SSM which is supposed to represent its physical mechanism is not even wrong. I have been living with a permanent shock for almost two decades. How can the world's leading scientific institutions still believe in the concepts of the SSM? The Sun is still a mystery based on its concepts, but a well known physicist who considers himself an authority, said in an interview that the current model made predictions that agreed with essentially all the observations to a pretty high level of accuracy. Absolutely ridiculous! In reality none of the basic features of the sun, without exception can be explained by the current model. The neutrino puzzle which is still alive is just one serious problem facing it. Furthermore, there is a long list of recent observations that have exposed entirely the dogmatic nature of the notion of thermonuclear furnace. However, these recent observations will be explained in other articles

\section{REFERENCES}

1- Experiments by R. Davis et al: see October 1990 Physics today, p 17.

2- Solar neutrino reviews: J. N. Bahcall and M. Pinsonneault, Reviews of Modern Physics, vol 64, p 885, 1992; S. Turck-Chieze and I. Lopes, Astrophysical Journal, vol 408, p 347, 1993. See also J. N. Bahcall, Neutrino Astrophysics (Cambridge, 1989).

3- Bahcall arguing for new physics: J. N. Bahcall and H. A. Bethe, Physical Review D, 47, p 1298, 1993; against new physics: J.N. Bahcall et al., Has a Standard Model Solution to the Solar Neutrino Problem Been Found?, preprint IASSNS-94/13 received at the National Radio Astronomy Observatory, 1994. J. Bahcall et al. Phys. Rev. Lett. 78, 171 (1997)

4- First results from the ${ }^{51} \mathrm{Cr}$ neutrino source experiment with the GALLEX detector, Physics Letters B, Volume 342, Issues 1-4, 12 January 1995, Pages 440-450, GALLEX Collaboration, P Anselmann, R Fockenbrock, W Hampel, G Heusser, J Kiko, T Kirsten, M Laubenstein, E Pernicka, S Pezzoni, U Rönn, M Sann, F Spielker, R Wink, M Wójcik, R.v Ammon, K.H Ebert, T Fritsch, D Heidt, E Henrich, C Schlosser, et al.

5- Constraints on Neutrino Oscillations Using 1258 Days of Super-Kamiokande Solar Neutrino Data, S. Fukuda et al. (Super-Kamiokande Collaboration) Phys. Rev. Lett. 86, 5656 - Published 18 June 2001

6- Mikheyev-Smirnov-Wolfenstein enhancement of oscillations as a possible solution to the solar-neutrino problem S. P. Rosen and J. M. Gelb Phys. Rev. D 34, 969 - Published 15 August 1986

7- High Energy Neutrino Production by Cosmic Ray Interactions in the Sun, G. Ingelman and M. Thunmana https://arxiv.org/pdf/hep-ph/9604288.pdf

8- Signatures of Cosmic Ray Interactions on the Solar Surface, Seckel, D., Stanev, T., \& Gaisser K. T Proceedings of the 21st International Cosmic Ray Conference Volume 4 (OG Sessions), p.463 Bibliographic Code: 1990ICRC....4..463S

\section{BIBLIOGRAPHY}

1- Are the experiments in error? Is our model of the solar interior wrong? http://helas.astro.uni.wroc.pl/data/jc/BS neutrino.ppt

2- Did the Nobel committee get the physics wrong? http://www.sciencemag.org/news/2016/12/did-nobel-committeeget-physics-wrong

3- $\quad$ The Sudbury Neutrino Observatory, https://arxiv.org/pdf/1602.02469.pdf

4- Sudbury Neutrino Observatory. https://en.wikipedia.org/wiki/Sudbury Neutrino Observatory

5- A half century with solar neutrinos. Nobel Lecture, December 8, 2002, by Raymond Davis, JR. https://www.nobelprize.org/nobel prizes/physics/laureates/2002/davis-lecture.pdf

6- Review of solar neutrino experiments. A. Bellerive. Ottawa-Carleton Institute for Physics, Department of Physics https://arxiv.org/pdf/hep-ex/0312045.pdf

7- John Bahcall's web site, http://www.sns.ias.edu/ jnb 
Author's Biography, Jamal S. Amar Shrair, PhD, Independent Scientist and Electrical Engineer Email: helical.cosmos@gmail.com , jamalshrair@yahoo.com

Website: www.helical-universe.info Phone: 0036309446992

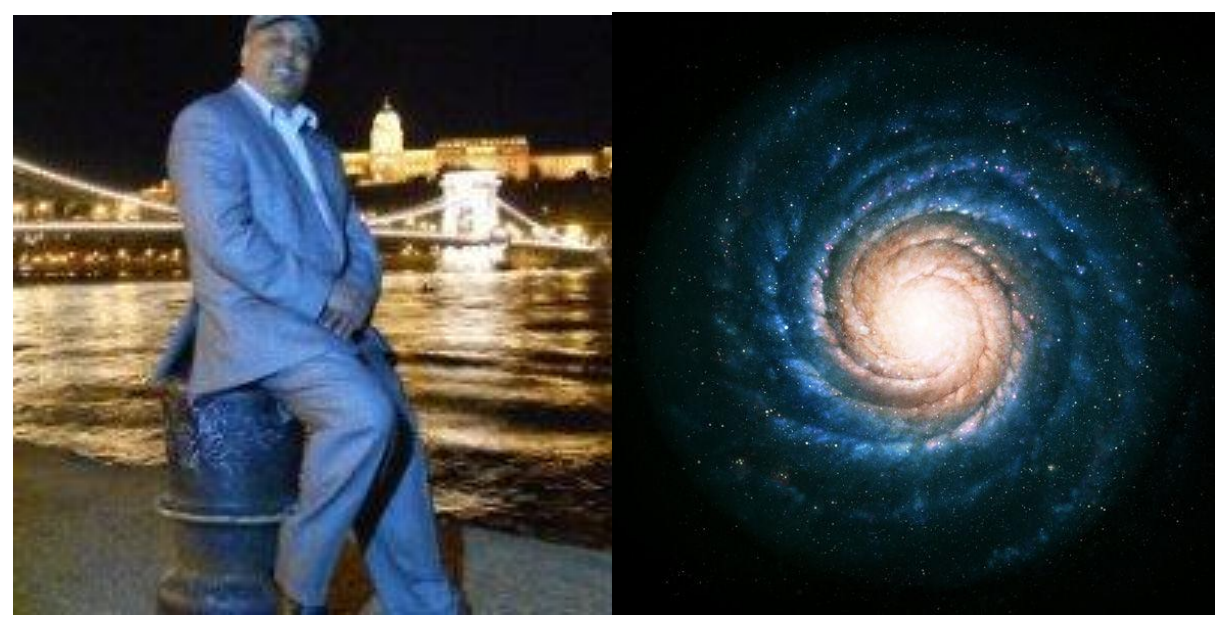

